\title{
Création d'un passeport vaccinal numérique contre le SRAS-CoV-2 au Canada
}

\author{
Kumanan Wilson MD MSc, Colleen M. Flood JSD
}

- Citation : CMAJ 2021 April 6;193:E486-8. doi : 10.1503/cmaj.210244-f; diffusion hâtive le 3 mars 2021

Voir la version anglaise de l'article ici : www.cmaj.ca/lookup/doi/10.1503/cmaj.210244

$\mathbf{L}$ a mise au point et le déploiement de vaccins sûrs et efficaces contre le coronavirus du système respiratoire aigu sévère 2 (SRAS-CoV-2) nous donnent l'espoir d'un allègement des lourdes conséquences sociales, économiques et sanitaires de la pandémie de maladie à coronavirus 2019 (COVID19). Les autorités publiques et les organisations privées pourraient bientôt exiger une preuve d'immunisation contre le SRAS-CoV-2 dans certains contextes, pour favoriser une relance en toute sécurité. Un « passeport vaccinal » ou un certificat ferait ainsi office de preuve de l'immunisation de son détenteur. Nous examinons ici les modalités d'application d'un passeport vaccinal contre le SRAS-CoV-2, l'infrastructure requise pour l'utiliser et les obstacles et limites potentiels de la méthode.

L'idée de fournir une preuve d'immunisation n'est pas nouvelle. Pendant les campagnes de vaccination antivariolique, la cicatrice de l'inoculation conditionnait souvent l'accès à certaines activités comme les voyages en train ${ }^{1}$. Au Canada, certaines provinces exigent des preuves d'immunisation pour l'inscription à l'école 2 . À l'échelle internationale, on exige une preuve de vaccination contre la fièvre jaune pour entrer dans les pays où la maladie est endémique, comme le prévoit l'annexe 7 du Règlement sanitaire internationa ${ }^{3,4}$. Dans le contexte actuel, la numérisation des carnets de vaccination et le passeport vaccinal numérique pour assurer l'accès sécuritaire aux activités et aux événements aux personnes démontrant leur immunité, constitue une première.

L'Organisation mondiale de la santé (OMS), l'Association du transport aérien international et le Forum économique mondial ont réfléchi aux normes et mécanismes potentiels pour la création d'un passeport vaccinal ${ }^{5-7}$, laissant entrevoir la probabilité qu'il serve en premier lieu pour les voyages internationaux. La présentation d'une preuve d'immunité pourrait aussi être étendue à certains lieux de travail et rassemblements de masse. Le passeport portant sur l'immunité naturelle a fait l'objet de réserves par le passé ${ }^{8}$. Nous pensons que l'utilisation du passeport concernera uniquement l'immunité conférée par les vaccins, car les preuves scientifiques à l'appui sont plus nombreuses que pour l'immunité naturelle.

\section{POINTS CLÉS}

- Les autorités publiques et les organisations privées pourraient bientôt exiger une preuve d'immunisation contre le coronavirus du syndrome respiratoire aigu sévère 2 (SRAS-CoV-2) dans certains contextes, pour favoriser une relance en toute sécurité.

- Les organismes internationaux ont étudié des normes et des solutions de passeport vaccinal potentielles en s'intéressant d'abord aux voyages internationaux.

- Au Canada, les gouvernements provinciaux et territoriaux doivent être en mesure de délivrer un carnet de vaccination numérique avec signature cryptographique à partir d'un registre gouvernemental afin que l'on puisse utiliser des passeports vaccinaux conformes aux normes nationales et aux initiatives internationales.

- Si les autorités gouvernementales n'implantent pas un passeport vaccinal, les sociétés privées comme les compagnies aériennes et les organisateurs de grands événements mettront sans doute en place leurs propres exigences et systèmes, ce qui risque de créer des problèmes d'équité, de protection de la vie privée et de coercition.

- Un accès limité aux vaccins, à la technologie et à d'autres ressources risque de faire obstacle à l'utilisation d'un passeport vaccinal.

Au Canada, le passeport vaccinal numérique contre le SRAS-CoV-2 pourrait prendre la forme d'un code à barres ou d'un code QR (Quick Response) dont la lecture indiquerait le statut vaccinal et qui serait stocké sur un appareil intelligent, comme une carte d'embarquement de compagnie aérienne. Le passeport, téléchargé à partir du registre de vaccination provincial ou territorial sur un portail gouvernemental, serait lié à l'identité d'une personne. Pour entrer dans un lieu ou participer à une activité à accès restreint, le titulaire du passeport présenterait le document au vérificateur, qui pourrait vérifier son statut immunitaire avec un lecteur. Le code à barres comprendrait une signature cryptographique attestant que le passeport a été délivré par une entité de confiance, comme un gouvernement provincial ou territorial. Le système de lecture devrait pouvoir vérifier si le produit utilisé, l'intervalle entre les doses et le nombre de doses 
satisfont les lignes directrices de santé publique en vigueur en ce qui concerne l'immunité, selon les données actuellement disponibles. Le titulaire du passeport pourrait également devoir fournir une pièce d'identité avec photo délivrée par les autorités.

Pour que le passeport vaccinal soit efficace, les gouvernements devront fixer plusieurs exigences fondamentales, comme celles de la Royal Society du Royaume-Uni (encadré 1$)^{9}$. Tout système doit se fonder sur un dossier de vaccination numérique exact et complet provenant d'un registre gouvernemental. Les gens doivent pouvoir consulter leur propre dossier et celui de leurs enfants et personnes à charge. Dans certaines provinces, des solutions d'identité numérique se dessinent pour permettre aux citoyens d'accéder en toute sécurité à leurs données de santé au moyen d'applications de fournisseurs externes, solutions qui pourraient servir pour le retour au travail. Les autorités publiques doivent être en mesure de produire et de délivrer des dossiers d'immunisation avec signature cryptographique. Il faut un logiciel, déployé de manière standardisée, pour vérifier le statut vaccinal et déterminer si le passeport répond aux exigences d'entrée ou d'accès en vigueur. Il faut également mettre en place des mécanismes de révocation des passeports, advenant que la recherche indiquerait une résistance des nouveaux variants du SRAS-CoV-2 aux vaccins. II sera difficile de déterminer quels paramètres de vaccination confèrent une immunité et la durée de celle-ci, car on sait qu'il y a déjà des écarts par rapport aux calendriers de vaccination recommandés.

L'élaboration et l'implantation d'un passeport vaccinal requièrent la collaboration d'experts des domaines juridique et éthique ${ }^{10}$. Il faut mettre en place des mesures de sécurité pour protéger la confidentialité des renseignements personnels, par exemple lors de la connexion de tiers au système pour vérifier le statut vaccinal. Il doit être possible de vérifier les informations contenues dans le passeport sans avoir à en faire la demande auprès de l'autorité émettrice. Toutefois, dans certains cas limites comme une révocation, le vérificateur se trouvera peutêtre à examiner la validité du passeport sur le serveur de l'émetteur, ce qui pourrait lui permettre de localiser le détenteur.

Encadré 1 : Critères de la Royal Society pour le passeport vaccinal ${ }^{9}$

- Satisfaire aux critères d'immunité contre le coronavirus du syndrome respiratoire aigu sévère 2

- Pouvoir intégrer les différences d'efficacité entre les vaccins et l'évolution de l'efficacité des vaccins contre les nouveaux variants

- Être uniforme à l'échelle internationale

- Contenir des sources vérifiables

- Avoir des utilisations définies

- Se fonder sur une plateforme de technologies interopérables

- Assurer la protection des données personnelles

- Être portable

- Être abordable pour les particuliers et les gouvernements

- Respecter les normes juridiques

- Respecter les normes éthiques

- Avoir des conditions d'utilisation comprises et acceptées par les détenteurs
Une autre préoccupation éthique importante concernant la mise en place d'un passeport vaccinal est l'accès aux vaccins. L'usage d'un tel passeport au Canada devrait rester limité jusqu'à ce que toute la population ait accès aux vaccins. D'ici là, dans certaines situations, les personnes seront tenues de présenter une preuve de vaccination ou une preuve récente de test de dépistage négatif du SRAS-CoV-2 pour faire une activité à accès restreint comme voyager en avion. Néanmoins, les personnes qui ne peuvent pas être vaccinées ne devraient pas faire l'objet de discrimination ou se voir refuser des services.

L'accès à la technologie risque aussi d'être un obstacle au passeport vaccinal. Les codes-barres ou les codes QR pourront probablement être imprimés, mais les personnes sans accès à des appareils numériques auront le désavantage de ne pouvoir consulter facilement les dossiers. L'obligation de présenter une preuve d'identité peut restreindre injustement l'accès des populations marginalisées qui ne disposent pas de cette preuve. Le développement technologique devrait se faire de concert avec un examen juridique et éthique afin que la solution choisie soit la façon la moins restrictive de déconfiner la société et ne nuise pas aux populations déjà marginalisées.

Au Canada, d'autres difficultés entravent la mise en place du passeport vaccinal numérique : elles sont liées à la maturité technologique, à l'absence d'identités numériques et à notre organisation politique en fédération. Le gouvernement fédéral devra examiner les normes internationales régissant les voyages internationaux, et éventuellement faciliter leur adoption par les gouvernements provinciaux à d'autres fins. Toujours au palier fédéral, le Comité consultatif national de l'immunisation devrait établir des normes sur ce qui constitue l'immunité conférée par vaccination. Comme l'immunisation est une compétence qui revient aux provinces et aux territoires, ceux-ci devraient mettre au point des dossiers d'immunisation numériques accessibles à partir de leurs registres d'immunisation en respectant autant que possible la vie privée et les autres droits de la personne. Toutefois, ces efforts à l'échelon provincial devront être encadrés par une norme fédérale ou internationale. Si les gouvernements ne se dotent pas des capacités nécessaires pour implanter le passeport vaccinal, il se peut que des sociétés privées (compagnies aériennes et organisateurs de grands événements) mettent en place leurs propres exigences et systèmes, ce qui risque de créer des problèmes d'équité, de protection de la vie privée et de coercition.

On s'attend au lancement imminent de passeports vaccinaux pour les voyages internationaux. Le Canada devra se conformer aux normes mondiales en matière de sécurité, d'authentification, de protection de la vie privée et d'échange de données élaborées dans le cadre de l'initiative du certificat de vaccination intelligent (Smart Vaccination Certificate) de ''OMS $^{5}$. Cette initiative bénéficierait en outre de la création de normes internationales sur ce qui constitue l'immunité et de la normalisation des approches de restriction d'accès selon l'évolution des données probantes en matière d'immunité. Une approche structurée d'implantation des passeports vaccinaux, testée d'abord pour les voyages internationaux, contribuerait à limiter la propagation du SRAS-CoV-2 tout en relançant l'économie mondiale. 


\section{Références}

1. Keelan J. Risk calculus and theories of viral attenuation in debates over compulsory smallpox vaccination in Montreal, 1870-1877. In: Kroker K, Keelan J, Mazumdar P, editors. Crafting immunity: working histories of immunological practice (History of medicine in context). Farnham (UK): Ashgate Publishing; 2008:29-54.

2. Immunization of School Pupils Act, R.S.O. 1990, c. I.1. Accessible ici : www. ontario.ca/laws/statute/90i01 (consulté le 27 janv. 2021).

3. Wilson K, Atkinson KM, Bell CP. Travel vaccines enter the digital age: creating a virtual immunization record. Am J Trop Med Hyg 2016;94:485-8.

4. International Health Regulations (2005) third edition. Geneva: World Health Organization; 2016. Accessible ici : www.who.int/publications/i/item/9789241580496 (consulté le 27 févr. 2021).

5. Smart Vaccination Certificate Working Group: about us. Geneva: World Health Organization. Accessible ici : www.who.int/groups/smart-vaccination -certificate-working-group (consulté le 27 janv. 2021).
6. IATA Travel Pass Initiative. Montréal: International Air Transport Association (IATA). Accessible ici : www.iata.org/en/programs/passenger/travel-pass/ (consulté le 27 janv. 2021).

7. CommonPass. The Commons Project. Accessible ici : https://thecommonsproject. org (consulté le 27 janv. 2021).

8. Kofler N, Baylis F. Ten reasons why immunity passports are a bad idea. Nature 2020;581:379-81.

9. Twelve criteria for the development and use of COVID-19 vaccine passports. The Royal Society; 2021 Feb. 14. Accessible ici : https://royalsociety.org/news/2021 /02/12-challenges-for-vaccine-passports/ (consulté le 20 févr. 2021).

10. Persad G, Emanuel EJ. The ethics of COVID-19 immunity-based licenses (“immunity passports"). JAMA 2020;323:2241-2.
Intérêts concurrents : Kumanan Wilson est président-directeur général de CANImmunize Inc. Cette société ne travaille pas sur un passeport vaccinal, mais on lui a demandé de s'exprimer sur la preuve du statut d'immunisation contre la COVID-19. CANImmunize a reçu des fonds provenant de sources publiques et privées (https://www.canimmunize.ca/fr/ partners). Le $D^{r}$ Wilson possède des actions de CANImmunize et ne reçoit aucune rémunération pour ses activités de PDG. Il est membre du comité indépendant sur la sécurité des données pour l'essai du vaccin de Medicago contre la COVID-19. Aucun autre intérêt concurrent n'a été déclaré.

Cet article a été révisé par des pairs.

Affiliations : Faculté de médecine (Wilson), École d'épidémiologie et de santé publique, Université d'Ottawa; Institut de recherche Bruyère et Institut de recherche de l'Hôpital d'Ottawa (Wilson); Faculté de droit (Flood), Section de common law, Université d'Ottawa; Centre de droit, politique et éthique de la santé de l'Université d'Ottawa (Flood), Ottawa, Ont.

Collaborateurs : Les deux auteurs ont contribué à la conception du travail, ont rédigé le manuscrit et en ont révisé de façon critique le contenu intellectuel important; ils ont donné leur approbation finale pour la version destinée à être publiée et assument l'entière responsabilité de tous les aspects du travail.
Propriété intellectuelle du contenu : Il s'agit d'un article en libre accès distribué conformément aux modalités de la licence Creative Commons Attribution (CC BY-NC-ND 4.0), qui permet l'utilisation, la diffusion et la reproduction de tout médium à la condition que la publication originale soit adéquatement citée, que l'utilisation se fasse à des fins non commerciales (c.-à-d., recherche ou éducation) et qu'aucune modification ni adaptation n'y soit apportée. Voir : https://creativecommons.org/ licenses/by-nc-nd/4.0/deed.fr.

Financement : Le financement pour cette recherche a été fourni en partie par le Groupe de travail sur l'immunité face à la COVID-19, créé par le gouvernement du Canada en collaboration avec les Instituts de recherche en santé du Canada.

Remerciements : Les auteurs remercient Cameron Bell pour ses conseils sur l'architecture technologique des passeports vaccinaux numériques et Bryan Thomas pour son apport sur le sujet.

Déclaration d'intérêts : Kumanan Wilson est marié à une employée du CMAJ, qui n'a pas participé au processus ayant mené au choix de cet article.

Correspondance : Kumanan Wilson, kwilson@ohri.ca 